\title{
LOCALIZED COLLOCATION TREFFTZ METHOD IN CONJUNCTION WITH THE GENERALIZED RECIPROCITY METHOD FOR TRANSIENT HEAT CONDUCTION ANALYSIS IN HETEROGENEOUS MATERIALS
}

\author{
ZHUOJIA FU ${ }^{1,2}$, MIN YANG $^{1}$, QIANG XI ${ }^{1} \&$ WENZHI XU ${ }^{1}$ \\ ${ }^{1}$ Center for Numerical Simulation Software in Engineering and Sciences, \\ College of Mechanics and Materials, Hohai University, China \\ ${ }^{2}$ State Key Laboratory of Mechanics and Control of Mechanical Structures, \\ Nanjing University of Aeronautics \& Astronautics, China
}

\begin{abstract}
This paper presents a novel localized collocation Trefftz method (LCTM) in conjunction with Laplace transformation for transient heat conduction analysis in heterogeneous materials under temperature loading. In contrast to the conventional CTM, the proposed LCTM divides the whole domain into many stencil support domains consisting of several discretization nodes. Inspired by the dual reciprocity method (DRM) and multiple reciprocity method (MRM), an efficient technique, the generalized reciprocity method (GRM), is proposed to derive the problem-dependent T-complete functions for approximating the particular solution of the nonhomogeneous heat conduction equations in the local subdomains. Based on the moving least square technique and T-complete functions, the LCTM numerical differentiation formulation at a certain node can be derived by using a linear combination of the T-complete functions at its adjacent discretization nodes in the related stencil support domain. It inherits the semi-analytical property from the conventional CTM and avoids the ill-conditioned dense matrix problem, which is present particularly in large-scale heat conduction analysis. Some numerical examples of heat conduction problems in heterogeneous materials are presented, and the numerical results demonstrate the accuracy and efficiency of the proposed LCTM in comparison with the known analytical solutions.
\end{abstract}

Keywords: T-complete functions, collocation methods, Laplace transformation, heat conduction, moving least square, dual reciprocity method, multiple reciprocity method.

\section{INTRODUCTION}

With the increasing demand for long-term service of equipment structures in hightemperature environments, numerical simulation plays an important role on the prediction of the heat conduction behaviors of the equipment structures [1]-[3]. Here we consider the following transient heat conduction equations with thermal loading $Q(\mathbf{x}, t)$ in heterogeneous materials,

$$
\begin{aligned}
\Re u(\mathbf{x}, t)=Q(\mathbf{x}, t), & \mathbf{x} \in \Omega, t \in[0, T], \\
\mathrm{B} u(\mathbf{x}, t)=g_{B}(\mathbf{x}, t), & \mathbf{x} \in \partial \Omega, t \in[0, T], \\
u(\mathbf{x}, 0)=g_{I}(\mathbf{x}), & \mathbf{x} \in \Omega,
\end{aligned}
$$


where $u(\mathbf{x}, t)$ denotes the temperature, $g_{B}(\mathbf{x}, t), g_{I}(\mathbf{x})$ stands for the prescribed boundary condition functions and initial condition functions, the differential operators $\mathfrak{R}=\sum_{i, j=1}^{2} \frac{\partial}{\partial x_{i}}\left(K_{i j} \frac{\partial}{\partial x_{j}}\right)-\rho c \frac{\partial}{\partial t}$ and $\mathrm{B}=p_{1}+p_{2} \sum_{i, j=1}^{2} K_{i j} \frac{\partial}{\partial x_{j}} n_{i}$, in which

$\mathrm{B}=\left\{\begin{array}{c}\text { Dirichlet }\left(\text { essential) boundary condition operator, when } p_{1}=1, p_{2}=0,\right. \\ \text { Neumann }\left(\text { natural) boundary condition operator, when } p_{1}=0, p_{2}=1,\right. \\ \text { Convective boundary condition operator, when } p_{1} \neq 0, p_{2} \neq 0,\end{array}\right.$

and

$\mathbf{K}=\left\{K_{i j}\right\}_{1 \leq i, j \leq 2}$ represents the thermal conductivity matrix, $\rho$ stands for the density, $c$ stands for the specific heat, $n_{i}$ denotes the components of the outward unit normal vector in the $x_{i}$-direction.

\section{METHODOLOGY}

This section introduces the numerical method used here to solve eqns (1)-(3), which includes Laplace transformation, localized collocation Trefftz method [4], [5], generalized reciprocity method and numerical inverse Laplace transformation [6]-[9].

\subsection{Laplace transformation}

Here Laplace transformation is used to convert eqns (1)-(3) to time-independent PDEs in Laplace-space domain. Let us set the definition of Laplace transformation as

$$
\mathfrak{I}^{(L)}(u(\mathbf{x}, t))=u^{(L)}(\mathbf{x}, p)=\int_{0}^{\infty} u(\mathbf{x}, t) e^{-p t} d t,
$$

then we get

$$
\begin{aligned}
& \mathfrak{R}^{(L)} u^{(L)}(\mathbf{x}, p)=Q_{g}^{(L)}(\mathbf{x}, p), \quad \mathbf{x} \in \Omega, \\
& \mathrm{B} u^{(L)}(\mathbf{x}, p)=g_{B}^{(L)}(\mathbf{x}, p), \quad \mathbf{x} \in \partial \Omega,
\end{aligned}
$$

where the transformed governing equation operator $\mathfrak{R}^{(L)}=\sum_{i, j=1}^{2} \frac{\partial}{\partial x_{i}}\left(K_{i j} \frac{\partial}{\partial x_{j}}\right)-\rho c p$ and the generalized source function $Q_{g}^{(L)}(\mathbf{x}, p)=-Q^{(L)}(\mathbf{x}, p)-\rho c g_{I}(\mathbf{x}), p$ stands for the Laplace transformation parameter, and the physical quantities in Laplace-space domain are represented by the superscript " $(L)$ ".

\subsection{Localized collocation solver}

This study employs a novel localized collocation solver for solving time-independent PDEs (5)-(6) in Laplace-space domain. In the localized collocation solver, the localized collocation Trefftz method (LCTM) in conjunction with generalized reciprocity method (GRM) is implemented, where the generalized reciprocity method (GRM) is inspired from the dual reciprocity method (DRM) [10] and multiple reciprocity method (MRM) [11] to approximate the particular solution of the nonhomogeneous PDEs in the local subdomains. When the T-complete function of the transformed governing equation operator 
$\mathfrak{R}^{(L)}=\sum_{i, j=1}^{2} \frac{\partial}{\partial x_{i}}\left(K_{i j} \frac{\partial}{\partial x_{j}}\right)-\rho c p$ at each discretization node can be obtained, the LCTM + GRM can be used directly. Otherwise, the analogy differential operator $\tilde{\mathfrak{R}}^{(L)}$ to $\mathfrak{R}^{(L)}$ should be determined according to the easy derivation of the related T-complete functions. Then eqn (5) can be rewritten as

$$
\tilde{\mathfrak{R}}^{(L)} \boldsymbol{u}^{(L)}(\mathbf{x}, p)=\tilde{Q}_{g}^{(L)}(\mathbf{x}, p), \quad \mathbf{x} \in \Omega,
$$

where $\tilde{Q}_{g}^{(L)}(\mathbf{x}, p)=Q_{g}^{(L)}(\mathbf{x}, p)+\left(\tilde{\mathfrak{R}}^{(L)}-\mathfrak{R}^{(L)}\right) u^{(L)}(\mathbf{x}, p)$.

By adopting Atkinson's splitting approach, the approximate solution of eqns $(5) /(7)$ and (6) can be expressed as

$$
u^{(L)}(\mathbf{x}, p)=u_{h}^{(L)}(\mathbf{x}, p)+u_{p}^{(L)}(\mathbf{x}, p)
$$

where $u_{h}^{(L)}(\mathbf{x}, p), u_{p}^{(L)}(\mathbf{x}, p)$ stands for the homogeneous and the particular solutions, respectively. Assuming that the particular solution $u_{p}^{(L)}(\mathbf{x}, p)$ satisfies

$$
\mathfrak{R}^{(L)} u_{p}^{(L)}(\mathbf{x}, p)=Q_{g}^{(L)}(\mathbf{x}, p) \text { or } \tilde{R}^{(L)} u_{p}^{(L)}(\mathbf{x}, p)=\tilde{Q}_{g}^{(L)}(\mathbf{x}, p),
$$

and then the homogeneous solution can be obtained by solving the following updated homogeneous equation

$$
\mathfrak{R}^{(L)} u_{h}^{(L)}(\mathbf{x}, p)=0 \quad \text { or } \tilde{\mathfrak{R}}^{(L)} u_{h}^{(L)}(\mathbf{x}, p)=0
$$

subjected to the updated boundary conditions

$$
\mathrm{B} u_{h}^{(L)}(\mathbf{x}, p)=g_{B}^{(L)}(\mathbf{x}, p)-\mathrm{B} u_{p}^{(L)}(\mathbf{x}, p)
$$

For each given $i$-th node $\mathrm{x}_{0}^{i}$, the related set of its $m$ nearest nodes $\left(\mathrm{x}_{1}^{i}, \mathrm{x}_{2}^{i}, \ldots, \mathrm{x}_{m}^{i}\right)$ around $\mathbf{x}_{0}^{i}$ can be found and named as a subdomain $\Xi_{i}$, and the center of this subdomain can be set as $\tilde{\mathrm{x}}^{i}=\frac{1}{m+1} \sum_{j=0}^{m} \mathrm{x}_{j}^{i}$. Then the generalized reciprocity method (GRM) introduces the associated differential operator $\mathfrak{R}_{1}^{(L)}$ to vanish the generalized source term $Q_{g}^{(L)}\left(\tilde{Q}_{g}^{(L)}\right)$ at each discretization node, namely,

$$
\begin{array}{r}
\mathfrak{R}_{1}^{(L)} \mathfrak{R}^{(L)} u_{p}^{(L)}(\mathbf{x}, p)=\mathfrak{R}_{1}^{(L)} Q_{g}^{(L)}(\mathbf{x}, p)=\left(\Delta-\frac{\Delta Q_{g}^{(L)}(\mathbf{x}, p)}{Q_{g}^{(L)}(\mathbf{x}, p)}\right) Q_{g}^{(L)}(\mathbf{x}, p)=0 \text { or } \\
\mathfrak{R}_{1}^{(L)} \tilde{\mathfrak{R}}^{(L)} u_{p}^{(L)}(\mathbf{x}, p)=\mathfrak{R}_{1}^{(L)} \tilde{Q}_{g}^{(L)}(\mathbf{x}, p)=\left(\Delta-\frac{\Delta \tilde{Q}_{g}^{(L)}(\mathbf{x}, p)}{\tilde{Q}_{g}^{(L)}(\mathbf{x}, p)}\right) \tilde{Q}_{g}^{(L)}(\mathbf{x}, p)=0
\end{array}
$$


where $\mathfrak{R}_{1}^{(L)}$ could be Laplace-type, Helmholtz-type and Modified-Helmholtz-type operators according to the value of $\frac{\Delta Q_{g}^{(L)}(\mathbf{x}, p)}{Q_{g}^{(L)}(\mathbf{x}, p)}\left(\frac{\Delta \tilde{Q}_{g}^{(L)}(\mathbf{x}, p)}{\tilde{Q}_{g}^{(L)}(\mathbf{x}, p)}\right)$.

Assuming that $\phi_{k}^{i}$ and $\varphi_{k}^{i}$ stands for the derived T-complete functions of $\mathfrak{R}_{1}^{(L)}$ and $\mathfrak{R}^{(L)}\left(\tilde{\mathfrak{R}}^{(L)}\right)$, the approximated formulation $u^{(L)}\left(\mathbf{x}_{j}^{i}, p\right)$ inside the related subdomain $\Xi_{i}$ can be represented by a linear combination of T-complete functions $\phi_{k}^{i}$ and $\varphi_{k}^{i}$ with unknown coefficients $\alpha_{k}^{i}$ and $\beta_{k}^{i}$

$$
u^{(L)}\left(\mathbf{x}_{j}^{i}, p\right)=\sum_{k=0}^{m} \phi_{k}^{i} \alpha_{k}^{i}+\sum_{k=0}^{m} \varphi_{k}^{i} \beta_{k}^{i} \text { with its matrix form } \mathbf{u}^{(L)}=\left[\begin{array}{ll}
\Phi^{i} & \Psi^{i}
\end{array}\right]\left[\begin{array}{l}
\boldsymbol{\alpha}^{i} \\
\boldsymbol{\beta}^{i}
\end{array}\right]=\boldsymbol{\Theta}^{i} \boldsymbol{\chi}^{i} .
$$

By employing the moving least square technique, the following function can be defined

$$
\Lambda\left(u^{(L)}\left(\mathbf{x}_{j}^{i}, p\right)\right)=\sum_{j=1}^{N}\left[\left(u^{(L)}\left(\mathbf{x}_{j}^{i}, p\right)-\mathbf{\Theta}^{i} \chi^{i}\right) w^{i}\left(d_{j}\right)\right]^{2},
$$

in which the weighting function $w^{i}\left(d_{j}\right)$ is defined as the following compact support quartic spline function given in the literature [12],

$$
w^{i}\left(d_{j}\right)=\left\{\begin{array}{l}
1-6\left(\frac{d_{j}}{d_{\max }}\right)^{2}+8\left(\frac{d_{j}}{d_{\max }}\right)^{3}-3\left(\frac{d_{j}}{d_{\max }}\right)^{4}, d_{j} \leq d_{\max }, \\
0, \\
d_{j}>d_{\max }
\end{array}\right.
$$

where $d_{\max }$ denote the maximum distance between central node $\tilde{\mathrm{x}}^{i}=\frac{1}{m+1} \sum_{j=0}^{m} \mathrm{x}_{j}^{i}$ and the nodes of its subdomain $\Xi_{i}$.

In order to get the LCTM approximated eqn (13), let us minimize the function $\Lambda\left(u^{(L)}\right)$ to determine the unknown coefficient $\chi^{i}$ and then generate the following linear equation system at each discretization node,

$$
\mathbf{D} \chi^{i}=\mathbf{b}_{R},
$$

where 


$$
\mathbf{D}=\left[\begin{array}{ccccc}
\sum_{j=1}^{N} \Theta_{j 1}^{2} w_{j}^{i} & \sum_{j=1}^{N} \Theta_{j 1} \Theta_{j 2} w_{j}^{i} & \sum_{j=1}^{N} \Theta_{j 1} \Theta_{j 3} w_{j}^{i} & \cdots & \sum_{j=1}^{N} \Theta_{j 1} \Theta_{j N} w_{j}^{i} \\
& \sum_{j=1}^{N} \Theta_{j 2}^{2} w_{j}^{i} & \sum_{j=1}^{N} \Theta_{j 2} \Theta_{j 3} w_{j}^{i} & \cdots & \sum_{j=1}^{N} \Theta_{j 2} \Theta_{j N} w_{j}^{i} \\
& & \sum_{j=1}^{N} \Theta_{j 3}^{2} w_{j}^{i} & \cdots & \sum_{j=1}^{N} \Theta_{j 3} \Theta_{j N} w_{j}^{i} \\
\vdots & & \ddots & \vdots \\
\mathbf{b}_{R} & =\left[\begin{array}{ccccc}
\Theta_{11} w_{1}^{i} & \Theta_{21} w_{2}^{i} & \cdots & \Theta_{N 1} w_{N}^{i} \\
\Theta_{12} w_{1}^{i} & \Theta_{22} w_{2}^{i} & \cdots & \Theta_{N 2} w_{N}^{i} \\
\vdots & \vdots & \ddots & \vdots \\
\Theta_{1 N} w_{1}^{i} & \Theta_{2 N} w_{2}^{i} & \cdots & \Theta_{N N} w_{N}^{i}
\end{array}\right],\left[\begin{array}{c}
u^{(L)}\left(\mathbf{x}_{1}^{i}, p\right) \\
u^{(L)}\left(\mathbf{x}_{2}^{i}, p\right) \\
\vdots \\
u^{(L)}\left(\mathbf{x}_{N}^{i}, p\right)
\end{array}\right]=\mathbf{b}_{D}\left(\mathbf{u}^{i}\right)^{(L)} .
\end{array}\right.
$$

Assuming that the matrix $\mathbf{D}$ is reversible, one can get

$$
\chi^{i}=\mathbf{D}^{-1} \mathbf{b}_{D}\left(\mathbf{u}^{i}\right)^{(L)}=\mathbf{W}\left(\mathbf{u}^{i}\right)^{(L)}
$$

Next substituting eqn (17) into eqn (13), the Laplace-space solutions at $\left(\mathbf{x}_{1}^{i}, p\right)$ can be represented as

$$
\left(u_{1}^{i}\right)^{(L)}=\Theta_{1}^{i} \mathbf{D}^{-1} \mathbf{b}_{D}\left(\mathbf{u}^{i}\right)^{(L)}=\sum_{j=1}^{N} W_{j}^{i}\left(u_{j}^{i}\right)^{(L)},
$$

where the weighting matrix $\mathbf{W}$ is a sparse matrix with $n s$ nonzero elements in one row, i.e. $\left\{\begin{array}{l}W_{j}^{i} \neq 0, \mathbf{x}_{j}^{i} \in \Xi_{i} \subset \Omega \\ W_{j}^{i}=0, \mathbf{x}_{j}^{i} \in \Omega \backslash \Xi_{i}\end{array}, n s\right.$ denotes the number of discretization nodes in the subdomain $\Xi_{i}$. Then eqns (5), (7) and (6) can be discretized as follows:

$$
\begin{aligned}
& \mathfrak{R}^{(L)} \sum_{j=1}^{N} W_{j}^{i} u^{(L)}\left(\mathbf{x}_{j}^{i}, p\right)=Q_{g}^{(L)}\left(\mathbf{x}_{j}^{i}, p\right), \\
& \text { or } \quad \tilde{\mathfrak{R}}^{(L)} \sum_{j=1}^{N} W_{j}^{i} u^{(L)}\left(\mathbf{x}_{j}^{i}, p\right)=\tilde{Q}_{g}^{(L)}\left(\mathbf{x}_{j}^{i}, p\right), \\
& \mathrm{B} \sum_{j=1}^{N} W_{j}^{i} u^{(L)}\left(\mathbf{x}_{j}^{i}, p\right)=g_{B}^{(L)}\left(\mathbf{x}_{j}^{i}, p\right), \quad \mathbf{x}_{j}^{i} \in \partial \Omega .
\end{aligned}
$$

Then the Laplace-space solutions $u^{(L)}\left(\mathbf{x}_{j}^{i}, p\right)$ can be obtained by solving the system of linear algebra eqns (19) and (20). 


\subsection{Numerical inverse Laplace transformation}

Here we introduce one of the well-established numerical inverse Laplace transformation algorithms, the fixed Talbot algorithm (FTA) [7], [8], to regain the time-dependent solution $u(\mathbf{x}, t)$ in the time-domain from the Laplace-space solutions $u^{(L)}\left(\mathbf{x}_{j}^{i}, p\right)$. The related computational formulation of fixed Talbot algorithm can be given as follow

$$
u\left(\mathbf{x}_{j}^{i}, T\right)=\frac{\bar{\xi}}{N_{L T}}\left\{\begin{array}{l}
\frac{1}{2} u^{(L)}\left(\mathbf{x}_{j}^{i}, \bar{\xi}\right) e^{\xi T} \\
+\sum_{j=1}^{N_{L T}-1} \operatorname{Re}\left[e^{T \omega\left(\eta_{j}\right)} u^{(L)}\left(\mathbf{x}, \hat{\omega}\left(\eta_{j}\right)\right)\left(1+\mathrm{i}\left(\bar{\zeta}\left(\eta_{j}\right)\right)\right)\right]
\end{array}\right\},
$$

where

$$
\begin{gathered}
\hat{\omega}\left(\eta_{j}\right)=\bar{\xi} \eta_{j}\left(\cot \eta_{j}+\mathrm{i}\right), \quad \bar{\zeta}\left(\eta_{j}\right)=\eta_{j}+\left(\eta_{j} \cot \eta_{j}-1\right) \cot \eta_{j}, \\
\bar{\xi}=\frac{2 N_{L T}}{5 T}, \quad \eta_{j}=\frac{j \pi}{N_{L T}}, \quad j=1,2, \cdots, N_{L T}-1 .
\end{gathered}
$$

For a specific time instant $T$, only $N_{L T}$ boundary value problems with the corresponding Laplace-transform parameter $p=\bar{\xi}$ and $\omega\left(\eta_{j}\right)$ are required to be solved. In this study, $N_{L T}=8$ is employed in the fixed Talbot algorithm.

\section{NUMERICAL RESULTS AND DISCUSSIONS}

This section presents the example to verify the efficiency of the proposed localized collocation Trefftz method (LCTM) in the solution of long-time heat conduction behaviour under heterogeneous materials. To measure the accuracy of the proposed LCTM, the relative error Rerr and L2 relative error Lerr are defined as follows,

$$
\begin{gathered}
\operatorname{Rerr}(u)=\left|\frac{u\left(\mathbf{x}^{i}, T\right)-u_{\text {exact }}\left(\mathbf{x}^{i}, T\right)}{u_{\text {exact }}\left(\mathbf{x}^{i}, T\right)}\right|, \\
\operatorname{Lerr}(u)=\sqrt{\frac{\sum_{i=1}^{N}\left(u\left(\mathbf{x}^{i}, T\right)-u_{\text {exact }}\left(\mathbf{x}^{i}, T\right)\right)^{2}}{\sum_{i=1}^{N} u_{\text {exact }}^{2}\left(\mathbf{x}^{i}, T\right)},}
\end{gathered}
$$

where $u\left(\mathbf{x}^{i}, T\right), u_{\text {exact }}\left(\mathbf{x}^{i}, T\right)$ stands for the numerical solutions and analytical solutions on the node $\mathbf{x}^{i}$ at the time instant $T$. Unless otherwise specified, the number of T-complete functions is chosen as $N_{T}=11$ and the k-nearest neighbors algorithm is used to select $m=12$ nearest nodes of the subdomain $\Xi_{i}$,

Here we consider the transient heat conduction problem in 2D square functionally graded material.

$$
\nabla(K(\mathbf{x}) \nabla u(\mathbf{x}, t))-\rho c \frac{\partial u(\mathbf{x}, t)}{\partial t}=Q(\mathbf{x}, t), \quad \mathbf{x} \in \Omega, t \in[0, T]
$$




$$
\begin{gathered}
\mathrm{B} u\left(x_{1}, x_{2}, t\right)=\mathrm{B}\left(l_{u}\left(x_{1}+x_{2}\right)+t\right), \quad \mathbf{x}=\left(x_{1}, x_{2}\right) \in \partial \Omega, t \in[0, T], \\
u\left(x_{1}, x_{2}, 0\right)=l_{u}\left(x_{1}+x_{2}\right), \quad \mathbf{x}=\left(x_{1}, x_{2}\right) \in \Omega,
\end{gathered}
$$

where the thermal conductivity $K(\mathbf{x})=\left(1+x_{1}+x_{2}\right)^{2}$, the thermal source loading $Q(\mathbf{x}, t)=\left(4 l_{u}-1\right)\left(1+x_{1}+x_{2}\right)$, the product of the density and the specific heat $\rho c=1+x_{1}+x_{2}$ . The analytical solution is $u\left(x_{1}, x_{2}, t\right)=l_{u}\left(x_{1}+x_{2}\right)+t$. By using Laplace transformation and variable transformation $v^{(L)}(\mathbf{x}, p)=\sqrt{K(\mathbf{x})} u^{(L)}(\mathbf{x}, p)$, the transient heat conduction problem can be converted to

$$
\begin{gathered}
(\Delta-p) v^{(L)}(\mathbf{x}, p)=Q_{g}^{(L)}(\mathbf{x}, p), \quad \mathbf{x} \in \Omega, \\
\mathrm{B} v^{(L)}\left(x_{1}, x_{2}, p\right)=\mathrm{B}\left(1+x_{1}+x_{2}\right)\left(l_{u}\left(x_{1}+x_{2}\right)+1 / p^{2}\right), \mathbf{x}=\left(x_{1}, x_{2}\right) \in \partial \Omega,
\end{gathered}
$$

where the generalized heat source $Q_{g}^{(L)}(\mathbf{x}, p)=\left(4 l_{u}-1-l_{u}\left(x_{1}+x_{2}\right)\right)\left(1+x_{1}+x_{2}\right)$. To vanish this generalized heat source term, the associated differential operator $\mathfrak{R}_{1}^{(L)}=\Delta-\frac{\Delta Q_{g}^{(L)}(\mathbf{x}, p)}{Q_{g}^{(L)}(\mathbf{x}, p)}=\Delta+\frac{4 l_{u}}{\left(4 l_{u}-1-l_{u}\left(x_{1}+x_{2}\right)\right)\left(1+x_{1}+x_{2}\right)}$ is implemented here. Then the related T-complete functions $\phi_{k}^{i}$ and $\varphi_{k}^{i}[13]$ can be derived as follows:

$$
\begin{gathered}
\phi_{k}^{i}=\left\{I_{0}\left(\kappa_{1} r_{k}^{i}\right), I_{1}\left(\kappa_{1} r_{k}^{i}\right) \cos \left(\theta_{k}^{i}\right), I_{1}\left(\kappa_{1} r_{k}^{i}\right) \sin \left(\theta_{k}^{i}\right), \ldots,\right. \\
\left.I_{N_{T} / 2}\left(\kappa_{1} r_{k}^{i}\right) \cos \left(N_{T} \theta_{k}^{i} / 2\right), I_{N_{T} / 2}\left(\kappa_{1} r_{k}^{i}\right) \sin \left(N_{T} \theta_{k}^{i} / 2\right)\right\} \\
\varphi_{k}^{i}=\left\{J_{0}\left(\kappa_{2} r_{k}^{i}\right), J_{1}\left(\kappa_{2} r_{k}^{i}\right) \cos \left(\theta_{k}^{i}\right), J_{1}\left(\kappa_{2} r_{k}^{i}\right) \sin \left(\theta_{k}^{i}\right), \ldots,\right. \\
\left.J_{N_{T} / 2}\left(\kappa_{2} r_{k}^{i}\right) \cos \left(N_{T} \theta_{k}^{i} / 2\right), J_{N_{T} / 2}\left(\kappa_{2} r_{k}^{i}\right) \sin \left(N_{T} \theta_{k}^{i} / 2\right)\right\},
\end{gathered}
$$

where $\kappa_{1}=\sqrt{p}$ and $\kappa_{2}=\sqrt{\frac{4 l_{u}}{\left(4 l_{u}-1-l_{u}\left(x_{1}+x_{2}\right)\right)\left(1+x_{1}+x_{2}\right)}}, r_{k}^{i}=\sqrt{\left(x_{k 1}^{i}-\tilde{x}_{1}^{i}\right)^{2}+\left(x_{k 2}^{i}-\tilde{x}_{2}^{i}\right)^{2}}$, $\theta_{k}^{i}=\arctan \left(\frac{x_{k 2}^{i}-\tilde{x}_{2}^{i}}{x_{k 1}^{i}-\tilde{x}_{1}^{i}}\right)$, in which $\mathrm{x}_{k}^{i}=\left(x_{k 1}^{i}, x_{k 2}^{i}\right), \tilde{\mathrm{x}}^{i}=\left(\tilde{x}_{1}^{i}, \tilde{x}_{2}^{i}\right)$.

Under several parameter settings, Table 1 presents the Lerr errors obtained by the proposed LCTM+FTA for the transient heat conduction problems with full Dirichlet BCs and mixed BCs in comparison with the exact Laplace-space solutions + FTA. In the proposed LCTM, 1020 uniform-distributed nodes are used. In the mixed BC cases, only the boundary $\left(x_{1}=0,0 \leq x_{2} \leq 1\right)$ is imposed on Neumann boundary condition, the remaining boundaries are imposed on Dirichlet boundary conditions. From Table 1, it can be found that with different parameter settings, the proposed LCTM + FTA provide the satisfactory numerical solutions for both the full Dirichlet BC cases and the mixed BC cases, which have a slight loss of accuracy in comparison with the retrieved time-dependent solutions from the exact Laplace- 
space solutions by fixed Talbot algorithm. It reveals that the numerical error is mainly

Table 1: Lerr errors obtained by the proposed LCTM + FTA for the transient heat conduction problems with full Dirichlet BCs and mixed BCs in comparison with the exact Laplace-space solutions + FTA under several parameter settings.

\begin{tabular}{|c|c|c|c|}
\hline \multirow{2}{*}{ Parameter setting } & \multicolumn{3}{|c|}{ Lerr errors } \\
\cline { 2 - 4 } & Full Dirichlet BCs & Mixed BCs & Exact Laplace-space solutions \\
\hline$l_{u}=0.1, T=10$ & $1.39 \mathrm{E}-2$ & $1.56 \mathrm{E}-2$ & $1.07 \mathrm{E}-2$ \\
\hline$l_{u}=0.1, T=100$ & $1.41 \mathrm{E}-3$ & $1.57 \mathrm{E}-3$ & $1.08 \mathrm{E}-3$ \\
\hline$l_{u}=0.1, T=500$ & $2.82 \mathrm{E}-4$ & $3.15 \mathrm{E}-4$ & $2.17 \mathrm{E}-4$ \\
\hline$l_{u}=0.01, T=10$ & $4.88 \mathrm{E}-3$ & $6.96 \mathrm{E}-3$ & $1.08 \mathrm{E}-3$ \\
\hline$l_{u}=0.01, T=100$ & $4.91 \mathrm{E}-4$ & $7.00 \mathrm{E}-4$ & $1.08 \mathrm{E}-4$ \\
\hline$l_{u}=0.01, T=500$ & $9.84 \mathrm{E}-5$ & $1.40 \mathrm{E}-4$ & $2.16 \mathrm{E}-5$ \\
\hline
\end{tabular}

appeared in the ill-posed NILT process. Moreover, the LCTM solutions for the full Dirichlet $\mathrm{BC}$ cases are slight accurate than the ones for the mixed BC cases. Fig. 1 displays the error distributions of the proposed LCTM+FTA for transient heat conduction problems with $l_{u}=0.01$ subjected to full Dirichlet BCs and mixed BCs at different time instants $(\mathrm{T}=10$, 100, 500). It can be observed from Fig. 1 that the maximum relative errors are appeared at the central region of the computational domains for full Dirichlet $\mathrm{BC}$ cases and the center region of the left boundary of the computational domains for mixed $\mathrm{BC}$ cases. Similar to the conclusions drawn from Table 1, the LCTM solutions for the full Dirichlet BC cases are slight accurate than the ones for the mixed BC cases.

\section{CONCLUSIONS}

This paper proposes a novel localized collocation solver based on the localized collocation Trefftz method (LCTM) in conjunction with fixed Talbot algorithm (FTA) and the generalized reciprocity method (GRM) for transient heat conduction analysis in heterogeneous materials under thermal loading. The transient heat conduction analysis in heterogeneous materials with arbitrary spatial variations can be carried out by implementing the proposed LCTM coupled with the GRM, where the proposed scheme provides more accurate and efficient solutions than those obtained by the traditional FEM due to the use of the semi-analytical T-complete functions, and it is available for transient heat conduction analysis in heterogeneous materials without the related derived T-complete functions. The satisfactory numerical solutions can be obtained by using the proposed LCTM + FTA, which have a slight loss of accuracy in comparison with the retrieved time-dependent solutions from the exact Laplace-space solutions by fixed Talbot algorithm. It reveals that the numerical error is mainly appeared in the ill-posed NILT process. Of course, it would be interesting to develop an appropriate NILT to make the proposed localized collocation solver more efficient for transient heat conduction analysis in heterogeneous materials under thermal loading, and investigates the efficiency of the proposed scheme for solving the transient heat conduction problems without the close-form analytical solutions, which are now under intense study. 


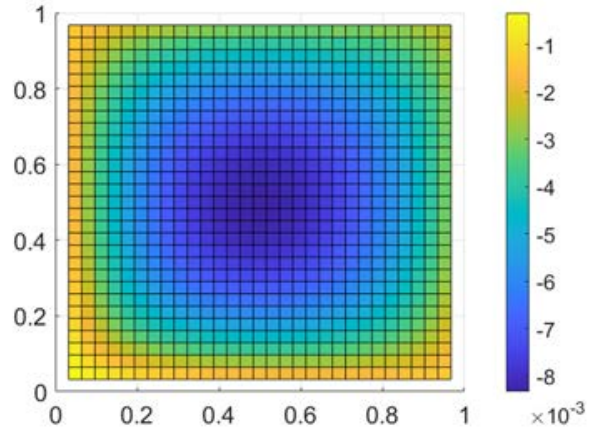

(a) Full Dirichlet BCs at $\mathrm{T}=10$

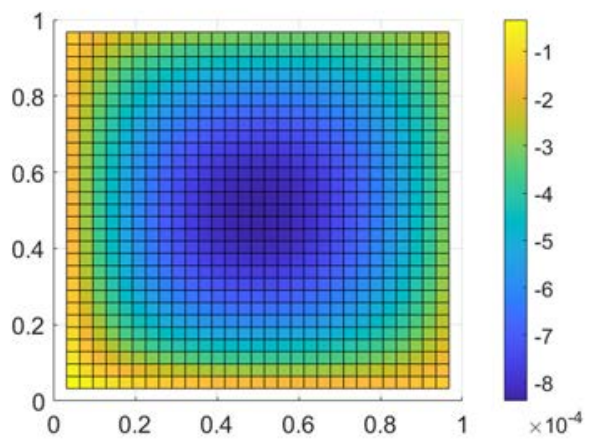

(c) Full Dirichlet BCs at $\mathrm{T}=100$

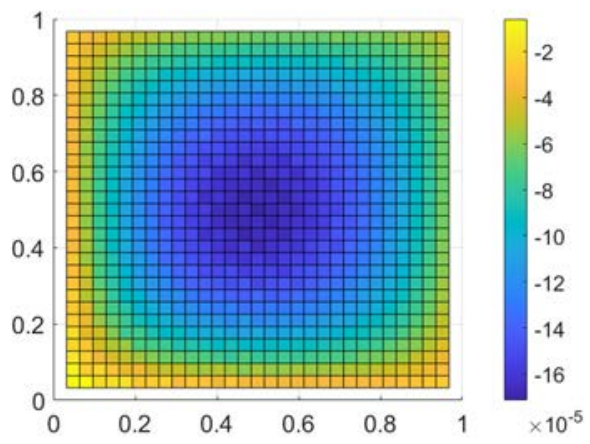

(e) Full Dirichlet BCs at $\mathrm{T}=500$

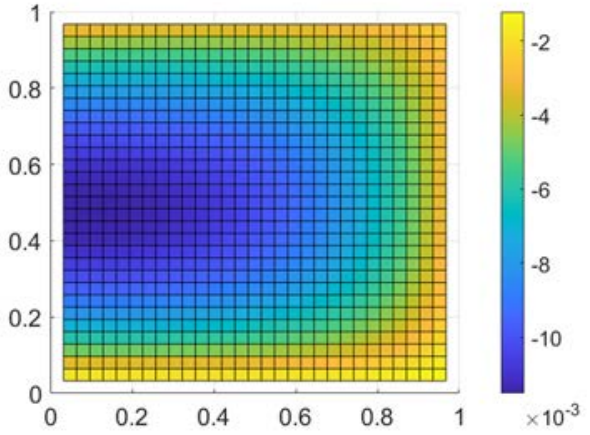

(b) Mixed BCs at $\mathrm{T}=10$

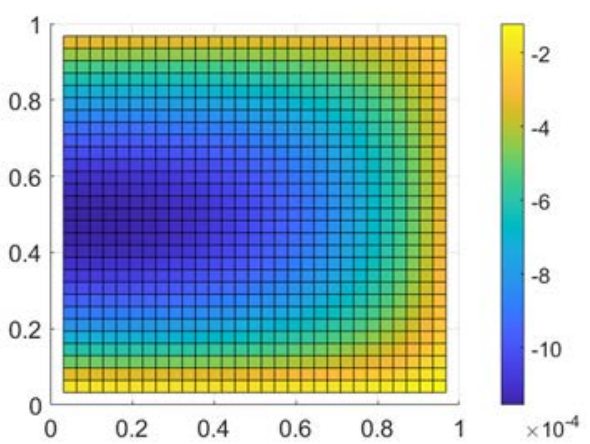

(d) Mixed BCs at $\mathrm{T}=100$

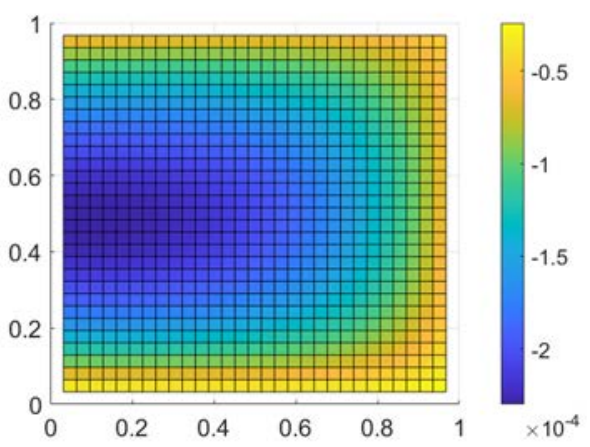

(f) Mixed BCs at $\mathrm{T}=500$

Figure 1: Error distributions of the proposed LCTM + FTA for transient heat conduction problems with full Dirichlet BCs and mixed BCs at different time instants $(\mathrm{T}=$ $10,100,500)$.

\section{ACKNOWLEDGEMENTS}

The work described in this paper was supported by the National Science Funds of China (Grant No. 11772119), the Fundamental Research Funds for the Central Universities (Grant No. B200202124), the Foundation for Open Project of State Key Laboratory of Mechanics and Control of Mechanical Structures (Nanjing University Of Aeronautics And Astronautics) 
(Grant No. MCMS-E-0519G01), and Six Talent Peaks Project in Jiangsu Province of China (Grant No. 2019-KTHY-009).

\section{REFERENCES}

[1] Malek, M., Izem, N. \& Seaid, M., A three-dimensional enriched finite element method for nonlinear transient heat transfer in functionally graded materials. International Journal of Heat and Mass Transfer, 155, p. 119804, 2020.

[2] Feng, W.Z. \& Gao, X.W., An interface integral equation method for solving transient heat conduction in multi-medium materials with variable thermal properties. International Journal of Heat and Mass Transfer, 98, pp. 227-239, 2016.

[3] Sladek, J., Sladek, V., Krivacek, J. \& Zhang, C., Local BIEM for transient heat conduction analysis in 3-D axisymmetric functionally graded solids. Computational Mechanics, 32(3), pp. 169-176, 2003.

[4] Xi, Q., Fu, Z., Wu, W., Wang, H. \& Wang, Y., A novel localized collocation solver based on Trefftz basis for potential-based inverse electromyography. Applied Mathematics and Computation, 390, p. 125604, 2021.

[5] Liu, Y.C., Fan, C.M., Yeih, W., Ku, C.Y. \& Chu, C.L., Numerical solutions of twodimensional Laplace and biharmonic equations by the localized Trefftz method. Computers \& Mathematics with Applications, 88, pp. 120-134, 2021.

[6] Sutradhar, A. \& Paulino, G.H., The simple boundary element method for transient heat conduction in functionally graded materials. Computer Methods in Applied Mechanics and Engineering, 193(42-44), pp. 4511-4539, 2004.

[7] Abate, J. \& Whitt, W., A unified framework for numerically inverting Laplace transforms. INFORMS Journal on Computing, 18(4), pp. 408-421, 2006.

[8] Fu, Z.J., Yang, L.W., Xi, Q. \& Liu, C.S., A boundary collocation method for anomalous heat conduction analysis in functionally graded materials. Computers \& Mathematics with Applications, 88, pp. 91-109, 2021.

[9] Fu, Z.J., Xi, Q., Chen, W. \& Cheng, A.H.D., A boundary-type meshless solver for transient heat conduction analysis of slender functionally graded materials with exponential variations. Computers \& Mathematics with Applications, 76(4), pp. 760773, 2018.

[10] Partridge, P.W., Brebbia, C.A. \& Wrobel, L.C., The Dual Reciprocity Boundary Element Method, Computational Mechanics Publications, 1992.

[11] Nowak, A.J. \& Neves, A.C., The Multiple Reciprocity Boundary Element Method, Computational Mechanics Publication, 1994.

[12] Gavete, L., Gavete, M.L. \& Benito, J.J., Improvements of generalized finite difference method and comparison with other meshless method. Applied Mathematical Modelling, 27(10), pp. 831-847, 2003.

[13] Li, Z.C., Lu, T.T., Huang, H.T. \& Cheng, A.H.D., Trefftz, collocation, and other boundary methods - A comparison. Numerical Methods for Partial Differential Equations: An International Journal, 23(1), pp. 93-144, 2007. 